\title{
Stimulus-Elicited Connectivity Influences Resting-State Connectivity Years Later in Human Development: A Prospective Study
}

\author{
Laurel Joy Gabard-Durnam, ${ }^{1 \star}$ Dylan Grace Gee, ${ }^{2 \star}$ Bonnie Goff, ${ }^{3}$ Jessica Flannery, ${ }^{4}$ Eva Telzer, ${ }^{5}$ \\ Kathryn Leigh Humphreys, ${ }^{3}$ Daniel Stephen Lumian, ${ }^{6}$ Dominic Stephen Fareri, ${ }^{1}$ Christina Caldera, ${ }^{3}$ \\ and Nim Tottenham ${ }^{1}$ \\ ${ }^{1}$ Department of Psychology, Columbia University, New York, New York 10025, ${ }^{2}$ Department of Psychiatry, Weill Cornell Medical College, New York, New \\ York 10065, ${ }^{3}$ Department of Psychology, University of California-Los Angeles, Los Angeles, California 90024, ${ }^{4}$ Department of Psychology, University of \\ Oregon, Eugene, Oregon 97403, ${ }^{5}$ Department of Psychology, University of Illinois at Urbana-Champaign, Champaign, Illinois 61801, and ${ }^{6}$ Department of \\ Psychology, University of Denver, Denver, Colorado 80208
}

Although the functional architecture of the brain is indexed by resting-state connectivity networks, little is currently known about the mechanisms through which these networks assemble into stable mature patterns. The current study posits and tests the long-term phasic molding hypothesis that resting-state networks are gradually shaped by recurring stimulus-elicited connectivity across development by examining how both stimulus-elicited and resting-state functional connections of the human brain emerge over development at the systems level. Using a sequential design following 4- to 18-year-olds over a 2 year period, we examined the predictive associations between stimulus-elicited and resting-state connectivity in amygdala-cortical circuitry as an exemplar case (given this network's protracted development across these ages). Age-related changes in amygdala functional connectivity converged on the same regions of medial prefrontal cortex ( $\mathrm{mPFC}$ ) and inferior frontal gyrus when elicited by emotional stimuli and when measured at rest. Consistent with the long-term phasic molding hypothesis, prospective analyses for both connections showed that the magnitude of an individual's stimuluselicited connectivity unidirectionally predicted resting-state functional connectivity 2 years later. For the amygdala-mPFC connection, only stimulus-elicited connectivity during childhood and the transition to adolescence shaped future resting-state connectivity, consistent with a sensitive period ending with adolescence for the amygdala-mPFC circuit. Together, these findings suggest that resting-state functional architecture may arise from phasic patterns of functional connectivity elicited by environmental stimuli over the course of development on the order of years.

Key words: amygdala; development; prefrontal cortex; prospective; resting-state connectivity; stimulus-elicited connectivity

\section{Significance Statement}

A fundamental issue in understanding the ontogeny of brain function is how resting-state (intrinsic) functional networks emerge and relate to stimulus-elicited functional connectivity. Here, we posit and test the long-term phasic molding hypothesis that resting-state network development is influenced by recurring stimulus-elicited connectivity through prospective examination of the developing human amygdala-cortical functional connections. Our results provide critical insight into how early environmental events sculpt functional network architecture across development and highlight childhood as a potential developmental period of heightened malleability for the amygdala-medial prefrontal cortex circuit. These findings have implications for how both positive and adverse experiences influence the developing brain and motivate future investigations of whether this molding mechanism reflects a general phenomenon of brain development.

\section{Introduction}

At the neural-systems level, the functional architecture of the brain is indexed by resting-state connectivity networks (Cole et

Received Feb. 23, 2016; revised March 15, 2016; accepted March 18, 2016.

Author contributions: L.J.G.-D., D.G.G., and N.T. designed research; L.J.G.-D., D.G.G., B.G., J.F., E.T., K.L.H., D.S.L., D.S.F., C.C., and N.T. performed research; L.J.G.-D. and D.G.G. analyzed data; L.J.G.-D., D.G.G., and N.T. wrote the paper. al., 2010; Raichle, 2010; Van Dijk et al., 2010; Pizoli et al., 2011). Accordingly, much recent work has focused on understanding resting-state network structure and function using fMRI 
(Bandettini and Smith, 2012). Resting-state networks show dramatic changes in composition across development (Jolles et al., 2011; Uddin et al., 2011; Barber et al., 2013; Solé-Padullés et al., 2016), but little is currently known about the mechanisms through which resting-state connections assemble into stable networks over time. Moreover, although the nature of restingstate connectivity differs from stimulus-elicited ("task-based") connectivity, the two measures often converge spatially and correlate with similar behavioral phenotypes in maturity (Cohen et al., 2005; Smith et al., 2009; Cox et al., 2010; Di et al., 2013; Mennes et al., 2013; MacNamara et al., 2015). However, the relation between stimulus-elicited and intrinsic resting-state connectivities is not yet well understood, and the interactions between these connectivities as they emerge over development has not been empirically examined. Characterizing the association between resting-state and stimulus-elicited connectivities prospectively as they mature across development is a powerful approach for identifying the mechanisms through which resting-state functional architecture arises and how resting-state connectivity may be interpreted in mature organisms.

A recent hypothesis suggests that mature resting-state functional architecture reflects the history of stimulus-elicited coactivations within a network (Miall and Robertson, 2006; Buckner and Vincent, 2007; Dosenbach et al., 2007; Mackey et al., 2013; Guerra-Carrillo et al., 2014). Here we specifically propose that, during development, accumulating experiences of phasic stimulus-elicited functional connectivity may gradually shape the formation of resting-state connectivity patterns (long-term phasic molding hypothesis). Phasic molding of functional architecture may be particularly pronounced during developmental periods when neural systems are most plastic and amenable to environmental inputs (Greenough et al., 1987). This mechanism of environmental inputs shaping the development of ongoing, resting activity has been demonstrated in the ferret visual cortex, where the activation patterns of neural populations at rest increasingly reflect that population's stimulus-evoked activity patterns over development (Berkes et al., 2011). The current study empirically tests whether the long-term phasic molding process occurs at the systems level in the developing human.

Specifically, amygdala-cortical networks are changing dramatically during childhood and adolescence, providing the opportunity to examine the long-term phasic molding hypothesis' set of predictions in the context of amygdala-cortical network maturation. In the present study, we used an emotional faces paradigm targeting amygdala-frontal connectivity as an exemplary network. We examined the amygdala-medial prefrontal cortex (mPFC) circuit a priori because we and others have reported extensive changes in this functional circuit and its associated emotion regulation behaviors across childhood and adolescence, facilitating examination of our hypothesis (Hare et al., 2008; Decety and Michalska, 2010; Perlman and Pelphrey, 2011; Gabard-Durnam et al., 2014; Gee et al., 2013; Swartz et al., 2014). We then conducted a whole-brain set of analyses with amygdala connectivity to test the specificity of the amygdalamPFC circuit findings. For the amygdala resting-state connections that were also modulated by the emotional faces stimuli, we

The authors declare no competing financial interests.

*L.J.G.-D. and D.G.G. contributed equally to this study.

Correspondence should be addressed to Dr. Laurel Joy Gabard-Durnam, Schermerhorn 409A, Department of Psychology, Columbia University, New York, NY 10025. E-mail: laurel.gabarddurnam@gmail.com.

DOI:10.1523/JNEUROSCI.0598-16.2016

Copyright $\odot 2016$ the authors $\quad 0270-6474 / 16 / 364772-14 \$ 15.00 / 0$
Table 1. Demographic information and participant characteristics for concurrent and prospective samples ${ }^{a}$

\begin{tabular}{lcl}
\hline & Cross-sectional sample & Prospective subsample \\
\hline$N$ & 53 & 23 \\
Age, years, mean (SD) & $13.4(4.9)$ & $11.3(3.8)$ \\
Sex & 27 male & 11 male \\
Handedness & 50 right & 22 right \\
IQ, mean (SD) & $114.5(18.0)$ & $117(19.8)$ \\
CBCL internalizing, mean (SD) & $5.1(5.2)$ & $4.3(3.6)$ \\
CBCL externalizing, mean (SD) & $5.1(4.6)$ & $5.1(5.1)$ \\
Income level, median (range) & $85,000-100,000$ & $85,000-100,000$ \\
& $(<10,000$ to $>200,000)$ & $(<10,000$ to $>200,000)$ \\
Ethnicity & & \\
$\quad$ African-American & $32 \%$ & $39 \%$ \\
$\quad$ Asian-American & $25 \%$ & $22 \%$ \\
$\quad$ American Indian & $6 \%$ & $9 \%$ \\
$\quad$ European-American & $55 \%$ & $57 \%$ \\
Other & $11 \%$ & $13 \%$ \\
$\quad$ Hispanic & $15 \%$ & $17 \%$ \\
\hline
\end{tabular}

${ }^{a} \mathrm{CBCL}$, Child Behavior CheckList.

tested a set of predictions. Based on the evidence in ferrets that stimulus-elicited and resting activity within a region are associated during development (Berkes et al., 2011), we anticipated that concurrent stimulus-elicited and resting-state connectivities would also be related within the same circuitry in humans. Moreover, consistent with our long-term phasic molding hypothesis of resting-state connectivity, we expected that stimulus-elicited connectivity would predict the nature of future resting-state connectivity during development. However, if resting-state connectivity reflects previous stimulus-elicited connectivity patterns, we hypothesized that resting-state connectivity would not predict the nature of future stimulus-elicited connectivity. To test these predictions, the present study used a prospective, sequential design to map both the concurrent cross-sectional and predictive relations between stimulus-elicited and resting-state connectivities within the developing amygdala functional network for youths from age 4 to 23 years.

\section{Materials and Methods}

Participants. Demographic information and participant characteristics for concurrent and prospective samples are presented in Table 1. Fiftythree participants contributed usable data for the cross-sectional analyses: 15 additional participants were excluded for either excessive motion during scanning (11 participants) or insufficient accuracy in the stimulus paradigm (4 participants); criteria described below; and 23 participants from the cross-sectional sample contributed usable data for the longitudinal analyses. Of the 53 participants' scans in the cross-sectional sample, 20 of these emotional faces task scans have previously been used in publication (Gee et al., 2013), and 48 of the resting-state scans have previously been used (Gabard-Durnam et al., 2014). However, comparisons between these scan types within individuals have never been published. None of the follow-up scans (scans 2 years later) have previously been used in any publication. These prior publications were used to define functional connections of interest, but the analyses and results reported here are entirely orthogonal to the prior publications. All participants were physically and psychiatrically healthy as confirmed by a telephone screening during recruitment and Child Behavior CheckList scores for internalizing and externalizing disorders (Table 1) (Achenbach, 1983). All participants had normal or corrected-to-normal vision. Prospective participants were excluded from participation if they had ever received a diagnosis of a learning or developmental disability, reported illicit drug use or alcohol use $>10$ drinks per week, or reported metal implants or any other contraindications to MRI. Adult participants were matriculated undergraduate students. This study was approved by the Institutional Review Boards of the University of California-Los Angeles and the 
state of California. All participants or their parents provided informed consent or assent (in the case of minors) for this study.

Procedure. In the first laboratory session, all participants were given the opportunity to acclimate to an MRI scanner environment with a mock MRI scanner. Recorded MRI noises were available for families to play at home to participants as further preparation. In the second session, participants returned to complete the first MRI scan with both the emotional faces stimuli and resting-state paradigms as part of a larger task battery (Telzer et al., 2013; Gee et al., 2013). To avoid capturing patterns of amygdala resting-state functional connectivity attributable to differences in initial MRI acclimation and arousal between the younger and older participants, all participants completed the resting-state scan at the end of the 45-min-long session. To preclude potential carryover effects from the fMRI stimuli, the resting-state scan was directly preceded by a buffer of $\sim 15 \mathrm{~min}$ of anatomical scans during which participants passively viewed a film. Although it is possible that the film viewing could influence subsequent amygdala activity, prior research has also shown that task ordering does not influence the amygdala-mPFC connectivity measures presently examined (Roy et al., 2009; Kim et al., 2011). Participant alertness was assessed through listening throughout the scan, direct observation at the end of the scan, and self-report of sleepiness. One older adolescent participant's data were discarded due to sleep during the resting-state scan. Two years after this first MRI session, participants completed a second MRI scan with both the emotional faces stimuli and resting-state paradigms in the same order as part of the larger task battery in the second scanning session.

fMRI paradigms. Participants completed two runs of an emotional faces paradigm that consisted of a mixed design with one blocked variable (emotional valence: happy vs fearful) and one event-related variable (emotional vs neutral). In each run, participants either viewed fearful faces interspersed with neutral faces or they viewed happy faces interspersed with neutral faces; the order of the fearful-neutral run and the happy-neutral run was counterbalanced across participants. Within each run, the stimuli were randomized and fixed across participants. To ensure that participants were paying attention to the stimuli, they were asked to press a button each time they saw a neutral face. Participants with accuracy rates $<50 \%$ on were excluded from further analysis $(4$ potential participants in the cross-sectional sample; these participants were not included in the demographic data for the usable participants). Female faces were selected from the Karolinska Directed Emotional Faces database (Lundqvist et al., 1998). The probability of a neutral face was $50 \%$ on any given trial. Stimuli were jittered (variable intertrial interval ranging from 3000 to $9000 \mathrm{~ms}$ ) and randomized based on a genetic algorithm (Wager and Nichols, 2003) to allow for unique estimates of the hemodynamic response for each trial type. Each run contained 48 trials (24 neutral faces, 24 fearful or happy faces). Each face was presented for $500 \mathrm{~ms}$. We examined the trials when participants passively viewed fearful faces or happy faces to assess stimulus-elicited connectivity (neutral faces were also included as a regressor in the model). The contrast of fear versus baseline and happy versus baseline allowed for the conditions to be matched on motor and attentional demands within the paradigm. Participants also completed a resting-state scan of 6 min duration in which they were instructed to lie still with their eyes closed (GabardDurnam et al., 2014).

All participants were scanned with a Siemens Trio 3.0-Tesla MRI scanner using a standard 12-channel radiofrequency head coil. For the emotional faces stimuli, we collected two functional scans. T2*-weighted echoplanar images (interleaved) were collected at an oblique angle of $\sim 15^{\circ}-30^{\circ}$ (selected per participant to minimize signal drop-out for their scans) ( 130 volumes/run; TR, $2000 \mathrm{~ms}$; TE, $30 \mathrm{~ms}$; flip angle, $90^{\circ}$; matrix size, $64 \times 64$; FOV, $192 \mathrm{~mm}$; 34 slices; $4 \mathrm{~mm}$ slice thickness; skip $0 \mathrm{~mm}$; 24 observations per event type). For the resting-state scan, we collected $\mathrm{T} 2^{\star}$-weighted echoplanar images at an oblique angle of $\sim 15^{\circ}-30^{\circ}(180$ volumes; TR, $2000 \mathrm{~ms}$; TE, $30 \mathrm{~ms}$; flip angle, $75^{\circ}$; matrix size, $64 \times 64$; FOV, $220 \mathrm{~mm}$; 33 slices; $4 \mathrm{~mm}$ slice thickness; skip $0 \mathrm{~mm}$ ). A wholebrain, high-resolution, T1-weighted anatomical scan (MP-RAGE; $192 \times$ 192 in-plane resolution, $256 \mathrm{~mm}$ FOV; $192 \mathrm{~mm} \times 1 \mathrm{~mm}$ sagittal slices) was acquired for each participant for registration and localization of functional data to Talairach space (Talairach and Tournoux, 1988).
Connections of interest identification. Conjunction analyses were performed with the amygdala resting-state connectivity and stimuluselicited connectivity analyses (contrast of fear vs baseline and happy vs baseline) reported previously (Gabard-Durnam et al., 2014 and Gee et al., 2013, respectively) to identify connections of interest showing spatial overlap across connectivities (whole-brain cluster corrected, $\alpha$ rate $<$ 0.05 ) for interrogation with the present sample's data. First, amygdala$\mathrm{mPFC}$ connections showing spatial overlap were determined for the agerelated changes in both stimulus-elicited connectivity and resting-state connectivity from the prior reports. An exploratory whole-brain conjunction of age-related changes across connectivities was then performed to identify any additional connections meeting this criterion that had not been previously examined (i.e., using the happy condition of stimuluselicited connectivity results). Next, any connections showing significant connectivity with the amygdala that did not change with age across both resting-state and stimulus-elicited connectivities were identified as positive control connections. These positive control connections facilitated testing whether amygdala connections that have already stabilized by this developmental period show associations between resting-state and stimulus-elicited connectivities, as predicted by the molding hypothesis. Last, connections showing age-related changes in amygdala resting-state connectivity but no significant age-related changes in either stimuluselicited connectivity condition (fear or happy) were identified as negative control connections. These negative control connections were used to test the prediction that for connections showing developmental (agerelated) change in resting-state connectivity that were not modulated by the demands of these particular stimuli, there should be no association between resting-state connectivity and either stimulus-elicited connectivity condition because this resting-state connectivity is shaped by other contexts (e.g., captured by other stimuli and paradigm designs).

Data preprocessing. The functional imaging data were preprocessed and analyzed with the Analysis of Functional NeuroImages (AFNI) software package (Cox, 1996). For each participant's images, preprocessing included discarding the first 4 functional volumes to allow for BOLD signal stabilization, correction for slice acquisition-dependent time shifts per volume, rigid-body translation and rotation from each volume to the first volume to generate 6 within-subject regressors, and spatial smoothing. Stimuli data were smoothed with a $6 \mathrm{~mm}$ isotropic FWHM kernel. Because the resting-state data had greater initial smoothness than the stimuli data, the resting-state data were smoothed to a $9 \mathrm{~mm}$ isotropic FWHM smoothness using 3dBlurToFWHM (i.e., various smoothing kernels were used across participants to achieve the same final smoothness of $9 \mathrm{~mm}$ ) to achieve equivalent effective spatial smoothness. To allow for comparisons across individuals for both stimuli and restingstate data, time courses were then normalized to percentage signal change, functional data were registered to the participant's anatomical scan, and the anatomical and functional scans were transformed to the standard coordinate space of Talairach and Tournoux (1988) with align_epi_anat.py. Transformations on the functional scans were combined into a single transformation within align_epi_anat.py to minimize the amount of interpolation applied to the functional data. Talairachtransformed images had a resampled resolution of $3 \mathrm{~mm}^{3}$. Both stimulus and resting-state data were processed using a bilateral, anatomical amygdala ROI defined in the Talairach atlas in AFNI (see Fig. 1, inset). Comparison of structural MRI and fMRI data between young children and adults by transformation to standard coordinate spaces, such as Talairach and Tournoux, has previously been shown to be methodologically appropriate (Burgund et al., 2002; Kang et al., 2003). Moreover, we have previously created anatomical averages for participants in this sample split into four age groups $(4-9,10-13,14-18$, and $19-23$ years) and overlaid these averages on the adult template to verify that amygdala seed regions in this developmental sample correspond to those in the adult template space after registration (Gabard-Durnam et al., 2014; Gee et al., 2013). The anatomical average from each of these developmental groups has coincided robustly with the adult template and with various anatomically defined amygdala regions, suggesting that registration of subcortical regions across development is not a confounding factor in this study.

Motion corrections. Consistent with recent recommendations, a strict motion-censoring limit was applied across stimuli and resting-state con- 
nectivity so that any time point and the immediately preceding time point were both censored if the Euclidean norm of the scan-to-scan motion parameters across the 6 rigid-body parameters exceeded $0.5 \mathrm{~mm} /$ degrees (mean length of retained data was $5.2 \mathrm{~min}$ of $6 \mathrm{~min}$ for the resting-state scan and $8.3 \mathrm{~min}$ of $8.7 \mathrm{~min}$ for the stimuli scan) (Siegel et al., 2014; Power et al., 2015). Given this motion restriction, five participants (ages 5, 6, 7, 13, and 14 years) contributed $<3.5$ min of usable resting-state data; however, because these participants were not outliers in any analysis and because resting-state correlation strengths have been shown to stabilize rapidly (Van Dijk et al., 2010), these participants' data were included in analyses. Nine participants were excluded from further analysis because $<3$ min of resting-state data meeting these motion criteria were obtained (from the $6 \mathrm{~min} \mathrm{scan}$ ), and two participants were excluded from any further analysis because $<4.4 \mathrm{~min}$ of stimuli data meeting these motion criteria were obtained (from the 8.7 min scan) (excluded participants' age range: $7-13$ years; these participants' data do not appear in the demographics or sample size reported for this study). Importantly, several recent reports have demonstrated that functional connectivity analyses are especially sensitive to motion artifacts (Satterthwaite et al., 2012; Van Dijk et al., 2012; Hallquist et al., 2013); thus, several further steps were taken to thoroughly address this potential confound.

For both the stimuli and the resting-state scans, at the within-subject level of analysis, 6 rigid-body motion regressors and the 6 backwards temporal derivatives of those regressors were included in all regressions to correct for head motion artifacts (Van Dijk et al., 2010; Yan et al., 2013). For the resting-state data, high-frequency signals have been shown to be most susceptible to motion confounds; thus, all data were temporally bandpass-filtered with a more conservative cutoff of $0.08 \mathrm{~Hz}$ (compared with the $0.1 \mathrm{~Hz}$ cutoff often used for resting-state data) as recommended by Satterthwaite et al. (2012). The mean framewise displacement (MFD) value was also calculated for each participant for both the stimuli and resting-state scans as described by Van Dijk et al. (2012). Control analyses were conducted at the group level with the MFD values entered as the regressor of interest in the previously published restingstate sample (Gabard-Durnam et al., 2014) and stimulus-elicited connectivity sample (Gee et al., 2013) to check that any significant motion-related effects did not overlap with the connections identified for use in the present analyses.

MFD values calculated for both the motion parameters for the restingstate and the stimuli data were also evaluated as potential covariates in the cross-sectional analyses. Neither the resting-state MFD nor the stimuli MFD values significantly correlated with their respective connectivity estimates, nor were they significant predictors of connectivity outcome measures in any analysis conducted (all $p>0.05$ ).

Stimulus-elicited connectivity statistical analysis. Psychophysiological interaction (PPI) analysis was conducted to assess stimulusdependent amygdala connectivity changes across the whole brain (Friston et al., 1997; Gee et al., 2013). To ensure that amygdala reactivity did not overly influence connectivity values, we controlled for amygdala reactivity at the trial level in this PPI analysis. A GLM analysis was performed in AFNI for each participant with regressors for stimuli, amygdala seed region time series, interaction of stimuli and time series, accuracy, time courses for eroded ventricle and eroded white matter masks as physiological nuisance covariates, and 12 motion regressors ( 6 rigid-body regressors and their 6 backwards temporal derivatives). Four psychological (stimuli) regressors modeled whether a given trial consisted of viewing an emotional face [i.e., fearful, happy, neutral faces (in the fearful run), and neutral faces (in the happy run)] or fixation. The physiological (seed region time series) regressor was the time series for the bilateral amygdala seed region after regressing out fixation and drift (by modeling linear and quadratic trends for the time series). Four interaction regressors modeled the interaction of the psychological regressors and the physiological regressor, such that each interaction regressor identified regions whose time series correlated in a stimulus-dependent manner with the amygdala time series. The GLM analyses fit the percentage signal change time series to each regressor, and linear and quadratic trends were modeled for the time series of each voxel to control for correlated drift.

Resting-state connectivity statistical analysis. For the resting-state data, time courses for eroded ventricle and eroded white matter masks and the global signal were extracted from the data. These time courses, along with 12 motion parameters ( 6 rigid-body motion parameters and their 6 backwards temporal derivatives), were simultaneously regressed out of the signal during temporal bandpass filtering $(0.009 \mathrm{~Hz}<\mathrm{f}<0.08 \mathrm{~Hz})$ as nuisance covariates to account for external contamination of the remaining resting-state frequencies (Power et al., 2015). (A secondary analysis processed the resting-state data without the global signal regressor, and connectivity estimates with and without global signal regression were very highly correlated for the a priori age-related connection of interest [Pearson's $r=0.94, n=53]$ ). Thus, all reported analyses were completed with the global signal regressor included to benefit from its reduction of motion and physiological artifacts (e.g., Chen et al., 2012; Keller et al., 2013; Power et al., 2015). Filtering was used to isolate the relevant signal fluctuations contributing to functional networks. An average time course for the bilateral amygdala seed region was then calculated after the combined filtering and nuisance regression of the data.

For each participant, a regression was performed using AFNI's 3 dREMLfit program to fit generalized Least Squares ARMA $(1,1)$ regression models correcting for temporal autocorrelation. Each regression model included the amygdala seed average time course. These regressions generated subject-level maps of the correlations between the amygdala time course and every other voxel's time course using the filtered, nuisance-regressed data.

Group-level analyses: stimulus-elicited baseline connectivity versus resting-state connectivity. The stimulus-elicited baseline condition modeled in the PPI is inherently different from the resting-state signal measured outside of the stimulus context, as the stimulus-elicited baseline is primarily indexing drift components explicitly regressed out of the resting-state signal. Resting-state data temporal filtering further limits the signal frequencies examined to those within the specific range of $0.009 \mathrm{~Hz}<\mathrm{f}<0.08 \mathrm{~Hz}$, while no filtering is applied to the stimuluselicited baseline data. Still, to ensure that the baseline condition of the stimulus-elicited connectivity did not significantly covary with restingstate functional connectivity, parameter estimates for the stimuluselicited baseline condition and the resting-state scan were extracted for the connections of interest in this study and compared across participants. As expected, Pearson correlations revealed no significant association between baseline and resting-state connectivity for any connection in this sample (all $p>0.25)$.

Cross-sectional analyses. Parameter estimates ( $\beta$-weights) of amygdala functional connectivity for the prespecified connections of interest for both stimuli (fear faces compared with baseline and happy faces compared with baseline) and resting-state paradigms from the subject-level analyses were then extracted and subjected to group-level regression analyses in SPSS (version 22). Any participants with parameter estimates $>3$ SDs away from the mean in either direction for stimulus or restingstate data were excluded as univariate overly influential outliers (a priori exclusion criteria). No participants were excluded from analyses in the cross-sectional sample using these criteria. Participants were removed as overly influential a priori multivariate outliers if they had Studentized deleted residuals, Standardized DFFITS, Standardized DFBETAS, and Covariance Ratio measure values outside of each of these measures' guidelines for small samples (a priori exclusion criteria to ensure no single data point could disproportionately enhance or confound results). Two participants were removed as overly influential multivariate outliers for all cross-sectional analyses, except the control analyses on connections with age-related changes in resting-state connectivity but not stimulus-elicited connectivity. In those control analyses, no participants were removed for one connection (parahippocampal $[\mathrm{PH}]$-amygdala connection), and four participants were removed for the other connection (superior temporal gyrus [STG]-amygdala connection). Partial correlation analyses were performed to examine whether stimulus-elicited and resting-state connectivities were associated for the connections of interest in this sample regardless of participants' ages. Effects of age, 
stimuli MFD value, and resting-state MFD value were therefore partialled out in the correlations.

Longitudinal analyses. The sample included for longitudinal analyses was the subsample of the cross-sectional participant sample who had stimuli and resting-state data from the first and second MRI scans. Parameter estimates of amygdala-PFC candidate connections and the two sets of control connections were extracted for stimulus-elicited and resting-state connectivity from both MRI visits and then subjected to group-level regression analyses in SPSS (version 22). No participants were removed from the sample as univariate overly influential outliers (a priori exclusion criterion). No participants were removed for the analyses of the candidate connection identified using the fear versus baseline stimulus condition as overly influential multivariate outliers (a priori exclusion criterion). Two participants were removed from the analyses of the candidate connection identified using the happy versus baseline stimulus condition as overly influential multivariate outliers (a priori exclusion criterion). For the set of analyses of the STG-amygdala control connection, one participant was removed from all analyses, except for the analysis of resting-state predicting stimulus-elicited connectivity to happy faces 2 years later, where two additional participants were removed as multivariate outliers (a priori exclusion criterion). For the set of analyses of the $\mathrm{PH}$-amygdala control connection, participants were removed as multivariate outliers only for the analyses of resting-state predicting stimulus-elicited connectivity 2 years later (three outliers for the happy-faces condition, one outlier for the fear-faces condition), and for the resting-state predicting resting-state connectivity 2 years later analysis (two outliers; a priori exclusion criterion). One participant was removed from the analyses of the medial frontal gyrus ( $\mathrm{mFG}$ )-amygdala control connection (a priori exclusion criteria).

In each longitudinal regression analysis, the time between the first and second MRI scans for each participant, centered to 2 years (the target scheduled time difference), was entered as a covariate to account for differences between participants in the timing of the two MRI sessions. The mean time difference between sessions for included participants was 1.8 years (SD 0.2 years; range 1.3-2.3 years). Participant's age was also included as a covariate in all analyses. Regressions were then performed predicting connectivity at the second MRI visit from the other type of connectivity measured at the first MRI visit (i.e., stimulus-elicited connectivity estimates predicting later resting-state connectivity estimates, and resting-state connectivity estimates predicting later stimulus-based connectivity estimates), with an interaction regressor coding an age by first visit connectivity estimate effect to examine developmental differences in the strength of this association. For nonsignificant interactions, the interaction regressor was removed from the model and regression was rerun to allow assessment of the main effects of the connectivity and age regressors. For significant interactions, post hoc simple slopes for the connectivity estimates were tested at the mean age (11.4 years), and $1 \mathrm{SD}$ above and below the mean age ( 15.2 and 7.5 years, respectively), which were also meaningful ages for this study sample as they indexed the interaction effect in different developmental periods (early childhood, the transition from childhood to adolescence, and mid-adolescence). Last, associations between a participant's connectivity parameter estimates at the first MRI visit and the second MRI visit were assessed within each connectivity type (e.g., association between resting-state parameter estimate at first MRI visit and second MRI visit) to complete the set of prospective analyses.

\section{Results}

Age-related amygdala-mPFC connection (resting-state + fear face-elicited connectivities): spatial overlap in resting-state and stimulus-elicited connectivities

The spatial overlap between amygdala-mPFC functional connections demonstrating age-related changes in both stimuluselicited (PPI contrast of fear vs baseline) (Gee et al., 2013) and resting-state connectivity (Gabard-Durnam et al., 2014) was first tested by calculating the statistical intersection between the connectivity results (whole-brain cluster corrected, $\alpha$ rate $<0.05$ ). There was significant spatial overlap in an amygdala-mPFC con- nection (Fig. 1A; 23 voxels, centered at $x=2, y=37, z=2$, Talairach atlas; Brodmann area 24). That is, developmental changes were identified within a similar amygdala-mPFC network emerging from both rest and stimulus-elicited data. This connection showing overlap in age-related change across both connectivity modalities served as the primary candidate connection for testing the molding hypothesis with cross-sectional and longitudinal analyses.

\section{Cross-sectional analysis: association between connectivities}

For the amygdala-mPFC candidate connection, the concurrent relation between stimulus-elicited and resting-state connectivities was then assessed within the cross-sectional sample from 4 to 23 years of age, over and above the effects of age, stimuli MFD values, and resting-state MFD values. Stimulus-elicited connectivity was inversely related to resting-state connectivity for this amygdala-mPFC connection, such that stronger (greater magnitude) negative stimulus-elicited amygdala-mPFC connectivity was associated with stronger positive resting-state amygdalamPFC connectivity (with the mature phenotype in young adults corresponding to negative stimulus-elicited connectivity and positive resting-state connectivity) (Pearson's $r$, partial $=$ $-0.302, p=0.037, n=51$; Fig. $1 B)$. That is, the different connectivity modalities were associated with each other when measured concurrently for the amygdala-mPFC connection where their age-related changes spatially overlap.

\section{Prospective analyses: stimulus-elicited connectivity predicts resting-state connectivity}

Next, to test the long-term phasic molding hypothesis, the prospective associations between the amygdala-mPFC connectivities were assessed across development through a series of predictive analyses using data from both the initial scan and the second scan 2 years later for participants $4-18$ years of age. First, regression was used to test whether stimulus-elicited connectivity measured at the first visit predicted resting-state connectivity 2 years later (main effect) and whether the strength of stimulus-elicited connectivity's effect differed across the ages in this sample (stimuluselicited connectivity by age interaction effect). This regression also controlled for the individual variability in the exact time difference between visits (centered to 2 years), the main effect of age at the first scan, and resting-state connectivity estimates from the first visit (to account for variance shared between resting-state and stimulus-elicited connectivity at the first visit). Consistent with the long-term phasic molding hypothesis, stimulus-elicited connectivity prospectively and inversely predicted resting-state connectivity 2 years later $(b$ coefficient $=$ $-0.042, t=-2.681, p=0.016, n=22$; Fig. $1 C$ ). However, this main effect was qualified by a significant interaction between age and stimulus-elicited connectivity at the first visit that predicted resting-state connectivity 2 years later $(b$ coefficient $=0.0002, t=$ $2.202, p=0.043, n=22$; Fig. 2A). This interaction effect suggests that stimulus-elicited connectivity shapes resting-state connectivity across time during specific developmental phases but not others (Fig. 2B).

Post hoc tests of simple slopes at ages 7.5, 11.4, and 15.2 years (mean $\pm \mathrm{SD}$ ) revealed that stimulus-elicited connectivity at the first visit at both 7.5 and 11.4 years of age significantly inversely predicted resting-state connectivity 2 years later (over and above the exact time difference between visits, age, and resting-state connectivity estimates from the first visit) (at age 7.5 years: $b$ coefficient $=-0.024, t=-3.033 p=0.008, n=22$; at age 11.4 years: $b$ coefficient $=-0.015, t=-3.259, p=0.005, n=22$; Fig. 

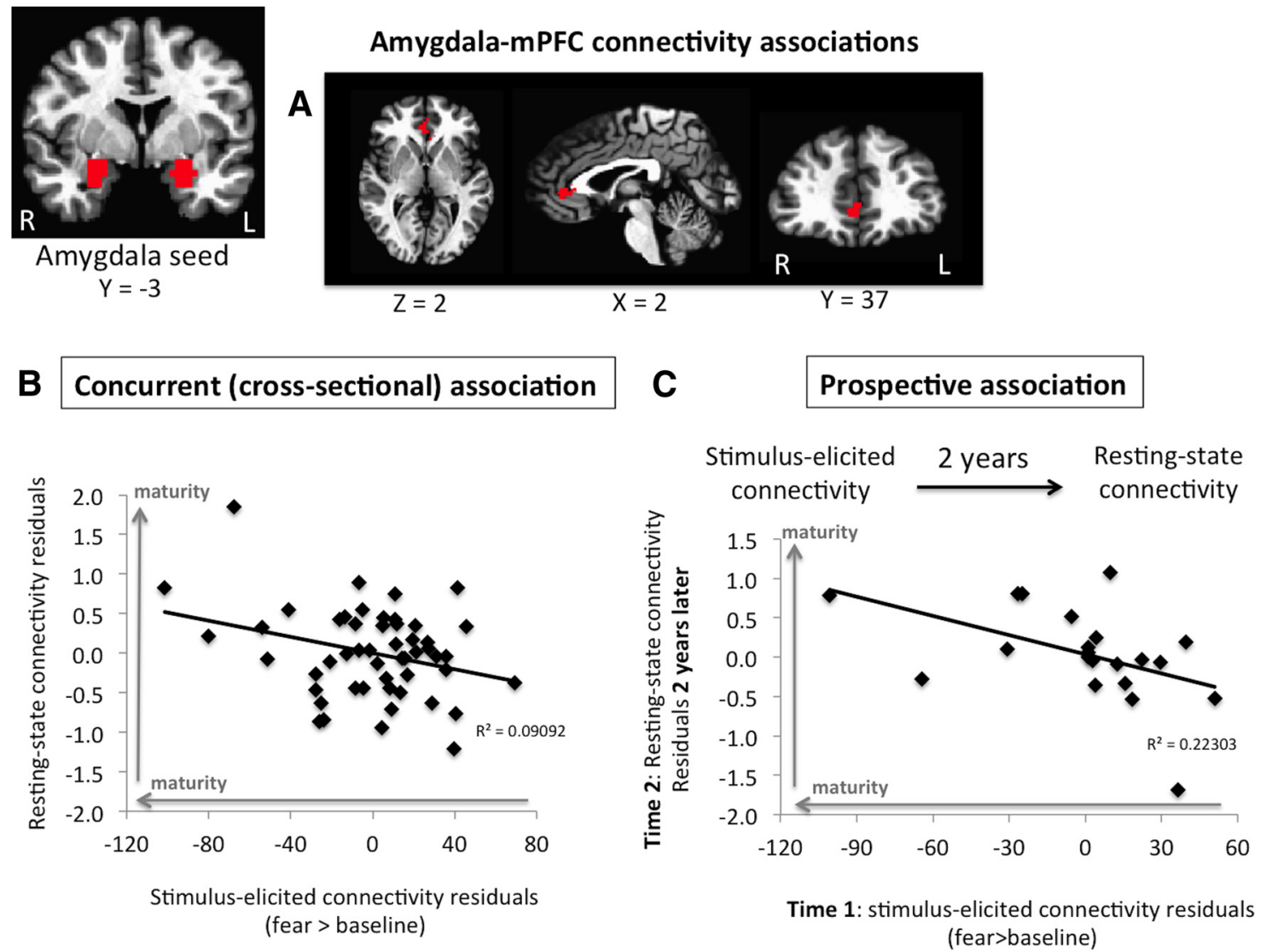

Figure 1. Amygdala-medial prefrontal cortex (mPFC) connection: connectivity associations. Inset, Bilateral anatomical amygdala seed region used to generate all connectivity for the study (Talairach atlas). $\boldsymbol{A}$, Area of mPFC overlap (Brodmann area 24) in age-related changes in stimulus-elicited (fear condition) and resting-state amygdala connectivity ( $p<0.05$, whole brain corrected) used to define amygdala-mPFC circuit. B, Concurrent association between residualized parameter estimates ( $\beta$ weights) for stimulus-elicited (fear condition) and resting-state functional connectivities, controlling for age and motion covariates. $\boldsymbol{C}$, Prospective association between residualized parameter estimates for stimulus-elicited (fear condition) connectivity measured at the first scan and resting-state functional connectivity measured at the second scan 2 years later, controlling for the exact time difference between scans, age, and resting-state connectivity estimates at the first scan.

$2 A)$. By contrast, stimulus-elicited connectivity at age $15.2 \mathrm{did}$ not predict resting-state connectivity 2 years later over and above the other variables in the model $(b$ coefficient $=-0.006$, $t=-1.545, p=0.142, n=22$; Fig. $2 A$ ). For both simple slopes at ages 7.5 and 11.4 years where the inverse association between stimulus-elicited and future resting-state connectivity was observed, the more negative the stimulus-elicited amygdala-mPFC connectivity at the first visit, the more positive the amygdala$\mathrm{mPFC}$ resting-state connectivity estimate was at the second visit 2 years later (Fig. 2A).

Prospective analyses: resting-state connectivity does not predict stimulus-elicited connectivity

In this same sample of participants, resting-state connectivity measured at the first visit did not predict the estimate of stimuluselicited connectivity 2 years later, nor was there a resting-state connectivity by age interaction in predicting future stimuluselicited connectivity (resting-state connectivity main effect: $b$ coefficient $=-20.95, t=-1.083, p=0.293, n=23$; restingstate $\times$ age interaction effect: $b$ coefficient $=-0.059, t=-0.149$, $p=0.884, n=23)$. This regression controlled for the individual variability in the exact time difference between scans, the effects of age at the first scan, and stimulus-elicited connectivity estimates from the first scan.
Prospective analyses: connectivities across 2 years

In addition to testing the long-term phasic molding hypothesis, associations across 2 years within each connectivity type were also assessed to complete the set of prospective analyses about connectivity associations. Stimulus-elicited amygdala-mPFC connectivity at the first visit did not significantly predict stimulus-elicited connectivity at the second visit 2 years later, controlling for the individual variability in the exact time difference between visits (Pearson's $r$, partial $=0.027, p=0.907, n=$ 22) (all connectivity associations summarized in Fig. 4). Restingstate connectivity at the first visit was associated with resting-state connectivity at the second visit at the trend level controlling for the same covariates (Pearson's $r$, partial $=0.401, p=0.08$, $n=22$ ) (see Fig. 4).

Additional age-related amygdala-PFC connectivity: amygdala-inferior frontal gyrus (IFG) connection (restingstate + happy face-elicited connectivities)/spatial overlap in resting-state and stimulus-elicited connectivities

A secondary exploratory analysis was conducted examining the whole-brain statistical conjunction of age-related changes in resting-state and stimulus-elicited connectivities (for the happy faces vs baseline condition) to identify any additional connections showing spatial overlap in age-related change that could be used to further test the molding hypothesis. An amygdala-IFG 


\section{Amygdala-mPFC connection: Effect of stimulus-elicited connectivity on future resting-state connectivity across development}

A

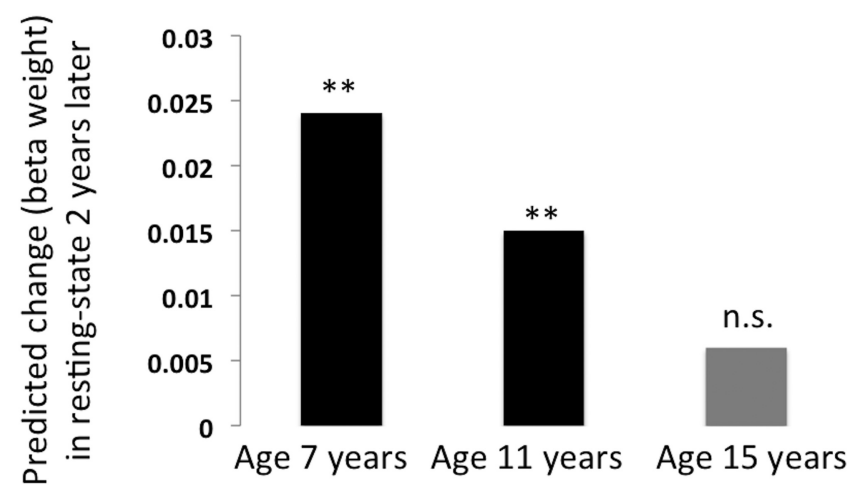

B

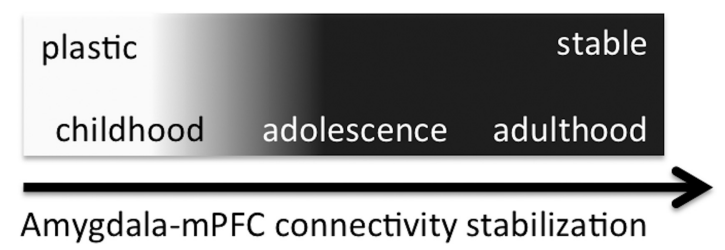

Figure 2. Amygdala-mPFC stimulus-elicited connectivity during childhood, but not adolescence, shapes future resting-state connectivity. A, Post hoc simple slopes evaluated at the mean age (11 years) \pm 1 standard deviation (7 years, 15 years) the prospective association between parameter estimates for stimulus-elicited (fear condition) connectivity measured at the first scan and resting-state functional connectivity measured at the second scan 2 years later. For each 1 unit increase in inverse (negative) stimulus-elicited connectivity (i.e., becoming increasingly negative) at age $7.5,11.4$, or 15.2 years, the expected increase in resting-state connectivity 2 years later is shown. ${ }^{* *} p<0.01$. n.s., Not significant. $\boldsymbol{B}$, Conceptual summary of the simple slopes tests.

connection (21 voxels, centered at $x=38, y=37, z=10$, Talairach atlas; Brodmann areas 46, 10) was identified with this whole-brain analysis (Fig. 3A). That is, developmental changes were identified within a similar amygdala-IFG network emerging from both rest and stimulus-elicited data. All analyses performed for the amygdala-mPFC connection were then repeated for this amygdala-IFG connection.

Cross-sectional analysis: association between connectivities First, the concurrent association between stimulus-elicited and resting-state connectivity for the amygdala-IFG connection in the cross-sectional sample was tested. A significant inverse association between resting-state and stimulus-elicited connectivities was found over and above age, stimulus-elicited MFD values, and resting-state MFD values (Pearson's $r$, partial $=-0.291, p=0.045, n=51$ ). More positive stimulus-elicited connectivity was associated with more negative resting-state connectivity concurrently (with the mature phenotype in young adults corresponding to positive stimuluselicited connectivity and negative resting-state connectivity) (Fig. 1B). That is, the different connectivity modalities were associated with each other concurrently for the amygdala-IFG connection where their age-related changes spatially overlap.

Prospective analyses: stimulus-elicited connectivity predicts resting-state connectivity

Given the concurrent association between connectivity modalities, prospective analyses were then conducted to test the long-term phasic molding hypothesis for the amygdala-IFG connection. First, regression tested whether stimulus-elicited connectivity measured at the first scan predicted resting-state connectivity 2 years later (main effect), and whether the stimulus-elicited connectivity's effect differed across age (interaction effect). The regression model covaried for individual variability in the time difference between visits, the main effect of age, and resting-state connectivity estimates from the first scan. Consistent with the molding hypothesis, a significant main effect of stimulus-elicited connectivity in predicting resting-state connectivity 2 years later was observed ((tested without the interaction term) $b$ coefficient $=-0.011, t=-2.226, p=0.042, n=20$; Fig. $3 B$ ). For this amygdala-IFG connection, the more positive the stimulus-elicited connectivity at the first visit, the more negative the resting-state connectivity estimate was at the second visit 2 years later. There was no significant interaction between stimulus-elicited connectivity and age in predicting resting-state connectivity 2 years later $(b$ coefficient $=0.560, t=0.597, p=0.56, n=20)$, suggesting that stimulus-elicited connectivity consistently shapes resting-state connectivity across childhood and adolescence for the amygdalaIFG connection.

Prospective analyses: resting-state connectivity does not predict stimulus-elicited connectivity

Consistent with the molding hypothesis, amygdala-IFG restingstate connectivity measured at the first scan did not predict the estimate of stimulus-elicited connectivity 2 years later over and above covariates' effects of time differences between visits, age, and stimulus-elicited connectivity from the first scan ( $b$ coefficient $=-51.42, t=-1.344, p=0.197, n=21$ ). There was no significant resting-state connectivity by age interaction in predicting future stimulus-elicited connectivity $(b$ coefficient $=$ $-0.600, t=-0.456, p=0.654, n=21$ ).

Prospective analyses: connectivities across 2 years

Associations across 2 years within each connectivity type were also assessed. Stimulus-elicited amygdala-IFG connectivity at the first visit did not significantly predict stimulus-elicited connec- 


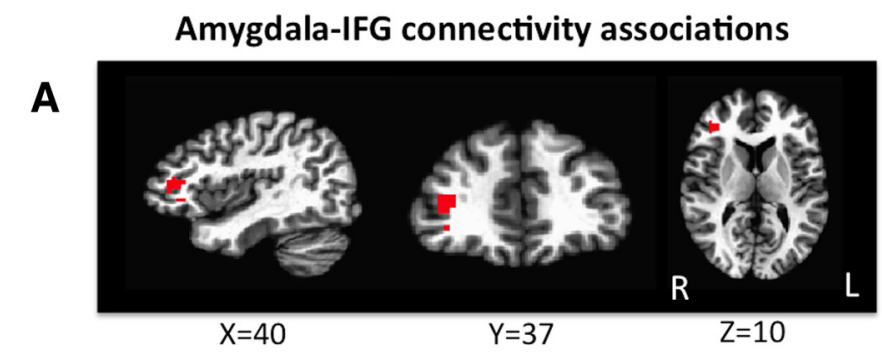

\section{B Concurrent (cross-sectional) association}

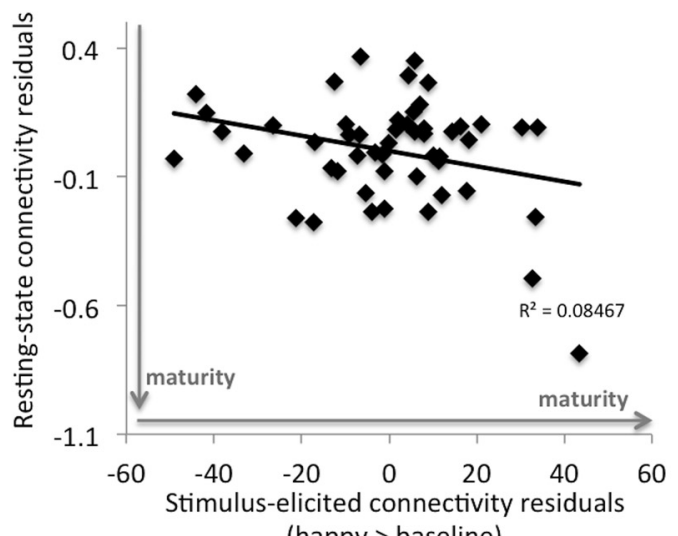

(happy > baseline)

\section{Prospective association}

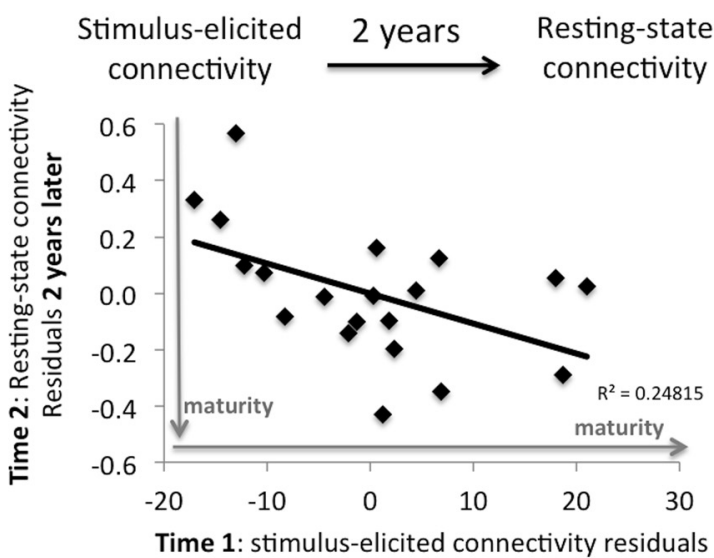

(happy>baseline)

Figure 3. Amygdala-inferior frontal gyrus (IFG) connection: connectivity associations. A, Area of IFG overlap (Brodmann areas 46, 10) in age-related changes in stimulus-elicited (happy condition) and resting-state amygdala connectivity ( $p<0.05$, whole brain corrected) used to define amygdala-IFG circuit. $\boldsymbol{B}$, Concurrent association between residualized parameter estimates ( $\beta$ weights) for stimulus-elicited (happy condition) and resting-state functional connectivities, controlling for age and motion covariates. $C$, Prospective association between residualized parameter estimates for stimulus-elicited (happy condition) connectivity measured at the first scan and resting-state functional connectivity measured at the second scan 2 years later, controlling for the exact time difference between scans, age, and resting-state connectivity estimates at the first scan.

\section{Scan 1:}

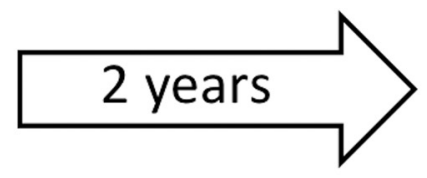

Scan 2:

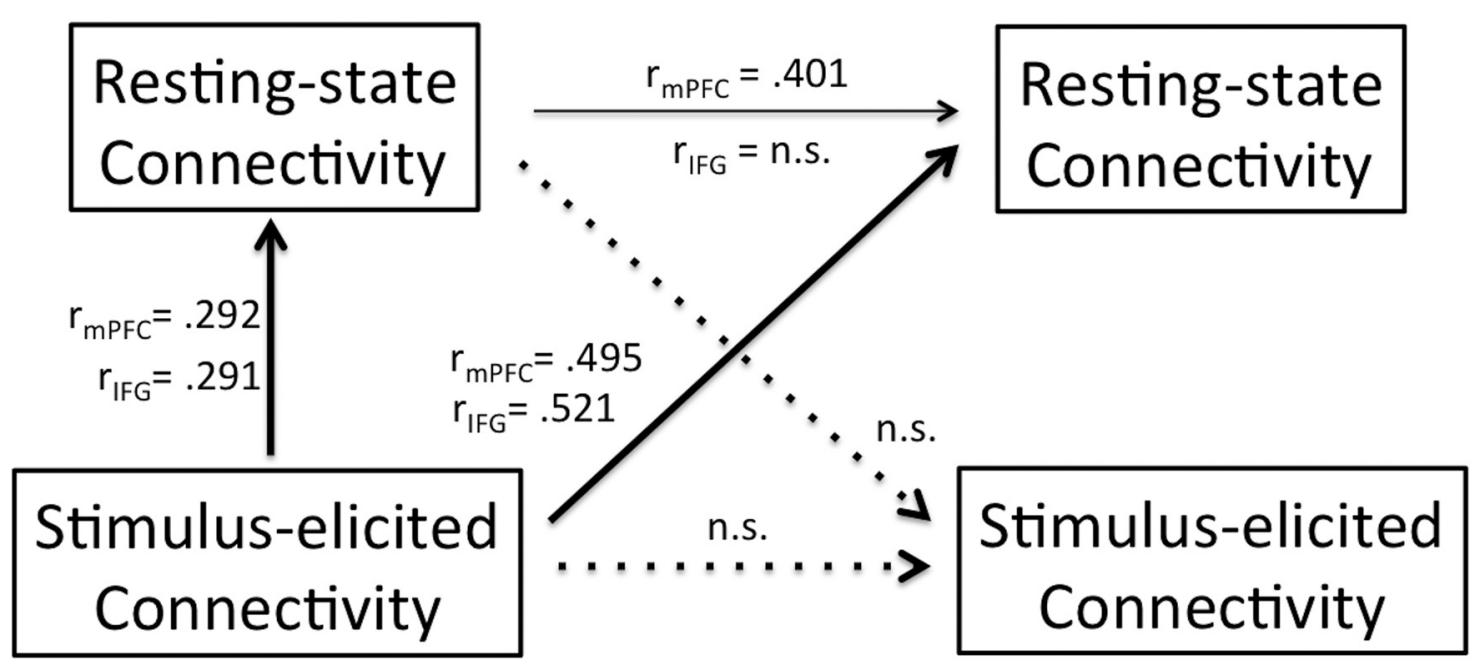

Figure 4. Concurrent and prospective connectivity associations within and between connectivity type. Graphic summarizing the complete set of concurrent and prospective associations (Pearson's $r$, partial) between stimulus-elicited and resting-state connectivities and prospective associations (Pearson's $r$, partial) within each connectivity type across childhood and adolescence. Dashed lines indicate nonsignificant (n.s.) associations. Thin solid lines indicate significant associations at the trend level $(p<0.1)$. Bold solid lines indicate significant associations at the level of $p<$ 0.05. Associations pertaining to the amygdala-mPFC connection elicited in the fear-face condition are indicated by the subscript " $\mathrm{PPFC}$," and associations pertaining to the amygdala-IFG connection elicited in the happy-face condition are indicated by the subscript "IFG." 


\section{Positive Control: Converging connectivity patterns with no age-related changes}

A

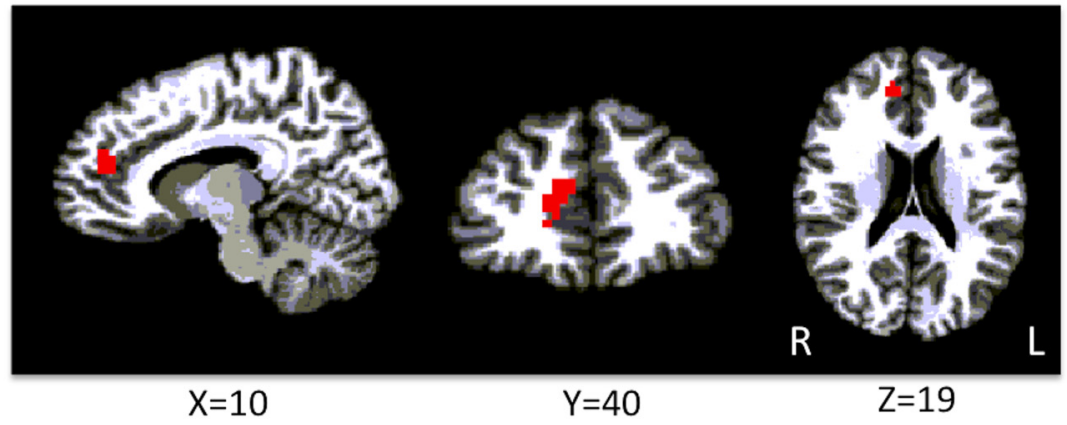

B

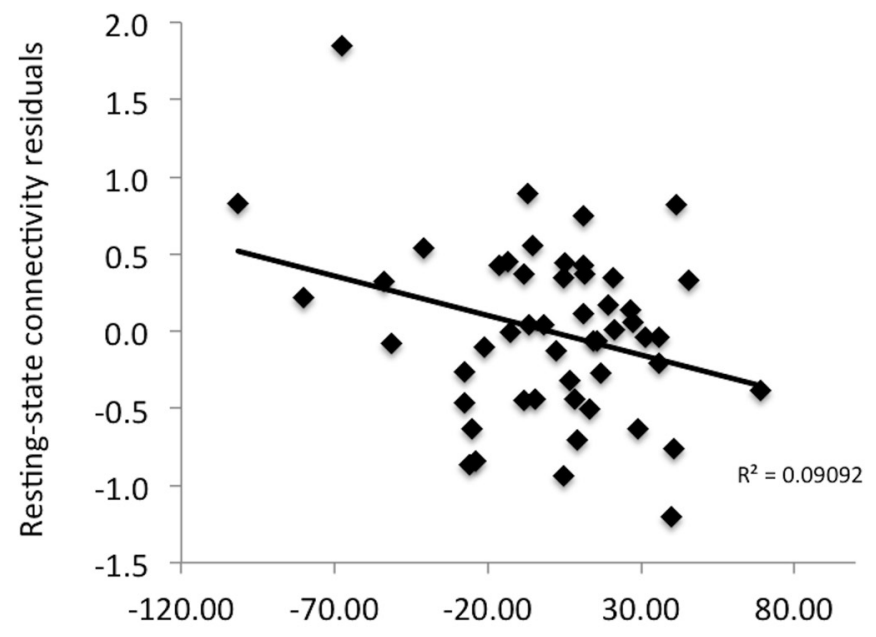

Stimulus-elicited connectivity

residuals (fear $>$ baseline)

Figure 5. Positive control. Amygdala-mFG: converging connectivity patterns with no age-related changes. $A$, Area of mFG overlap (Brodmann areas $9,10,32$ ) in significant stimulus-elicited (fear condition) and resting-state amygdala connectivity with no age-related changes ( $p<0.05$, whole brain corrected) used to define amygdala-mFG circuit. $\boldsymbol{B}$, Concurrent association between residualized parameter estimates ( $\beta$ weights) for stimulus-elicited (fear condition) and resting-state functional connectivities, controlling for age and motion covariates.

tivity at the second visit 2 years later, controlling for the individual variability in the exact time difference between visits and the effects of age (Pearson's $r$, partial $=0.013, p=0.955, n=21$ ) (Fig. 4). Resting-state connectivity at the first visit was associated with resting-state connectivity at the second visit at the trend level over and above those same covariates (Pearson's $r$, partial $=$ 0.080, $p=0.737, n=21$ ) (Fig. 4).

Control connections without convergent age-related changes Last, two whole-brain statistical conjunction analyses were performed to identify two additional connection types that did not exhibit convergent age-related changes in connectivity for control analyses related to the long-term phasic molding hypothesis.

Positive control connection: converging connectivity patterns with no age-related changes

First, if stimulus-elicited connectivity shapes the developmental change in resting-state connectivity as the long-term phasic molding hypothesis predicts, then stimulus-elicited and restingstate connectivities would also be expected to show an association with each other for stabilized connections that result from the molding process. Therefore, as a positive control analysis, associations were tested between concurrent resting-state and stimulus-elicited connectivity for stable amygdala connections. Connections showing significant connectivity with the amygdala that did not change with age (4-23 years) across both restingstate and stimulus-elicited connectivities were identified as stable control connections. This statistical conjunction analysis identified an amygdala-mFG connection (fear vs baseline condition with resting-state, 32 voxels, centered at $x=12, y=42, z=17$, Brodmann areas 9, 10, 32; Fig. 5A). Consistent with the prediction about stable connectivities, a significant inverse association was found between resting-state and stimulus-elicited connectivity for this amygdala-supragenual mFG connection when measured concurrently, controlling for age and MFD covariates (Pearson's $r$, partial: $-0.335, p=0.020, n=51$; Fig. $5 B$ ). Across participants, more positive resting-state connectivity was associated with more negative stimulus-elicited connectivity for the amygdala-mFG connection. In the smaller prospective subsample of participants, no significant association was observed between connectivities (Pearson's $r$, partial: $-0.165, p=0.474$, $n=22$ ).

Negative control connections: nonconvergence of age-related changes in connectivity patterns

Second, connections showing age-related changes in restingstate connectivity that were not modulated by the emotional faces paradigm (i.e., did not show significant stimulus-elicited connectivity or age-related changes in stimulus-elicited connectivity) would not be expected to show associations between 
this paradigm's stimulus-elicited connectivity estimates and resting-state connectivity concurrently or prospectively. That is, the resting-state connectivity may be shaped by other contexts that the emotional faces paradigm does not capture for this set of connections.

\section{Cross-sectional control analyses: no associations between connectivities}

To perform a control analysis testing the prediction that stimuluselicited and resting-state connectivity would show no association concurrently for the negative control connections, a statistical conjunction analysis identified connections showing age-related changes in resting-state connectivity but no significant age-related changes in or significant age-constant stimulus-elicited connectivity in any condition (fear or happy). Two such negative control connections met these criteria: a right hemisphere amygdala-STG connection (285 voxels, centered at $x=49, y=-32, z=11$, Talairach atlas; Brodmann areas 41, 21; Fig. $6 A$ ) and a bilateral amygdala-PH connection (216 voxels, centered at $x=23,-41,-3$ (right hemisphere), $x=-28, y=-39, z=-8$ (left hemisphere), Talairach atlas; Brodmann area 36; Figure 6D). Consistent with the prediction, neither connection showed significant associations between the stimuluselicited connectivity in either happy or fear conditions and the resting-state connectivity estimate when measured concurrently, over and above the effects of age and MFD values (amygdala-STG fear condition and resting-state: Pearson's $r$, partial $=-0.241, p=$ $0.11, n=49$; amygdala-STG happy condition and resting-state: Pearson's $r$, partial $=0.081, p=0.59, n=49$; Figure $6 B$; amygdala-PH fear condition and resting-state: Pearson's $r$, partial $=$ $-0.180, p=0.21, n=53$; amygdala-PH happy condition and resting-state: Pearson's $r$, partial $=-0.082, p=0.57, n=53$; Figure $6 E$ ).

\section{Prospective control analyses: stimulus-elicited connectivity does not predict resting-state connectivity}

Next, a prospective analysis tested the prediction that stimuluselicited connectivity would not prospectively predict later restingstate connectivity for the amygdala-STG and amygdala-PH control connections (i.e., no long-term molding of resting-state connectivity for the negative control connections). Regression models for each connection tested whether stimulus-elicited connectivity measured at the first scan (a model for the fear condition and a separate model for the happy condition) predicted resting-state connectivity 2 years later, covarying for individual variability in the time difference between visits, the main effect of age, and resting-state connectivity estimates from the first scan. Consistent with the prediction, no effect of stimulus-elicited connectivity in predicting resting-state connectivity 2 years later was observed for either condition (fear or happy) for either connection (amygdala-STG fear condition and resting-state: $b$ coefficient $=0.001, t=-0.606, p=0.553, n=21$; amygdala-STG happy condition and resting-state: $b$ coefficient $=$ $-0.002, t=-0.671, p=0.512, n=21$; Fig. $6 C$; amygdala-PH fear condition and resting-state: $b$ coefficient $=0.003, t=0.944, p=$ $0.359, n=22$; amygdala-PH happy condition and resting-state: $b$ coefficient $=0.005, t=1.36, p=0.192, n=22$; Fig. $6 F$ ).

\section{Prospective control analyses: resting-state connectivity does not predict stimulus-elicited connectivity}

Moreover, as expected for both fear- and happy-face conditions, across both control connections, resting-state connectivity measured at the first scan did not predict the estimate of stimuluselicited connectivity 2 years later either, over and above covariates' effects of time differences between visits, age, and stimulus-elicited connectivity from the first scan (amygdala-STG fear condition and resting-state: $b$ coefficient $=21.365, t=0.787$, $p=0.442, n=22$; amygdala-STG happy condition and restingstate: $b$ coefficient $=20.350, t=1.076, p=0.299, n=20$; amygdala-PH fear condition and resting-state: $b$ coefficient $=$ $-25.817, t=-1.283, p=0.217, n=22$; amygdala-PH happy condition and resting-state: $b$ coefficient $=47.407, t=1.558, p=$ $0.140, n=20)$.

\section{Prospective analyses: control connections' connectivities across 2 years}

These connections were identified as having significant agerelated changes in resting-state connectivity (but no significant stimulus-elicited connectivity), so only associations across 2 years within the resting-state connectivity type were assessed. For the amygdala-STG control connection, resting-state connectivity at the first visit was negatively associated with resting-state connectivity at the second visit 2 years later at the trend level over and above the effect of age and controlling for individual variability in the time difference between visits (Pearson's $r$, partial $=-0.416$, $p=0.077, n=21$ ), consistent with the age-related change from positive to negative connectivity previously observed for this connection (Gabard-Durnam et al., 2014). For the amygdala-PH control connection, resting-state connectivity at the first visit was positively associated with resting-state connectivity at the second visit 2 years later at the trend level over and above the same covariates (Pearson's $r$, partial $=0.428, p=0.077, n=20$ ).

\section{Discussion}

A fundamental issue in understanding the ontogeny of brain function is how resting-state functional networks emerge and relate to stimulus-elicited functional connectivity. Here, we posit and test the long-term phasic molding hypothesis that restingstate network development is influenced by recurring stimuluselicited connectivity through prospective examination of the developmental precursors leading to stable mature human functional connectivity for amygdala-cortical connections. These findings show that the developmental emergence of a restingstate network is indeed heavily influenced by the nature of previous phasic stimulus-elicited connectivity.

To test the long-term phasic molding hypothesis, we focused primarily on the amygdala-mPFC circuit known to show robust developmental changes during this period and to ultimately assemble stable connectivity in maturity supporting emotion regulation behavior (Banks et al., 2007; Roy et al., 2009; Perlman and Pelphrey, 2011; Gabard-Durnam et al., 2014; Gee et al., 2013; Swartz et al., 2014). We found that the age-related changes in resting-state and stimulus-elicited functional connectivities colocalized spatially; that is, the same $\mathrm{mPFC}$ region showed agerelated changes in connectivity with the amygdala across both measures. The stimulus-elicited and resting-state connectivities demonstrated highly related but opposite connectivity valences for this circuit when measured concurrently. Critically, during childhood and the transition to adolescence, stimulus-elicited connectivity measured at baseline predicted resting-state connectivity measured 2 years later (over and above baseline restingstate connectivity), explaining approximately one-fourth of the variance in the later resting-state estimates. However, there was no reciprocal developmental influence of baseline resting-state connectivity on stimulus-elicited connectivity measured 2 years later. These findings were replicated for an amygdala-IFG functional connection identified from a whole-brain analysis, with the exception that stimulus-elicited amygdala-IFG connectivity was predictive of future resting-state connectivity across child- 

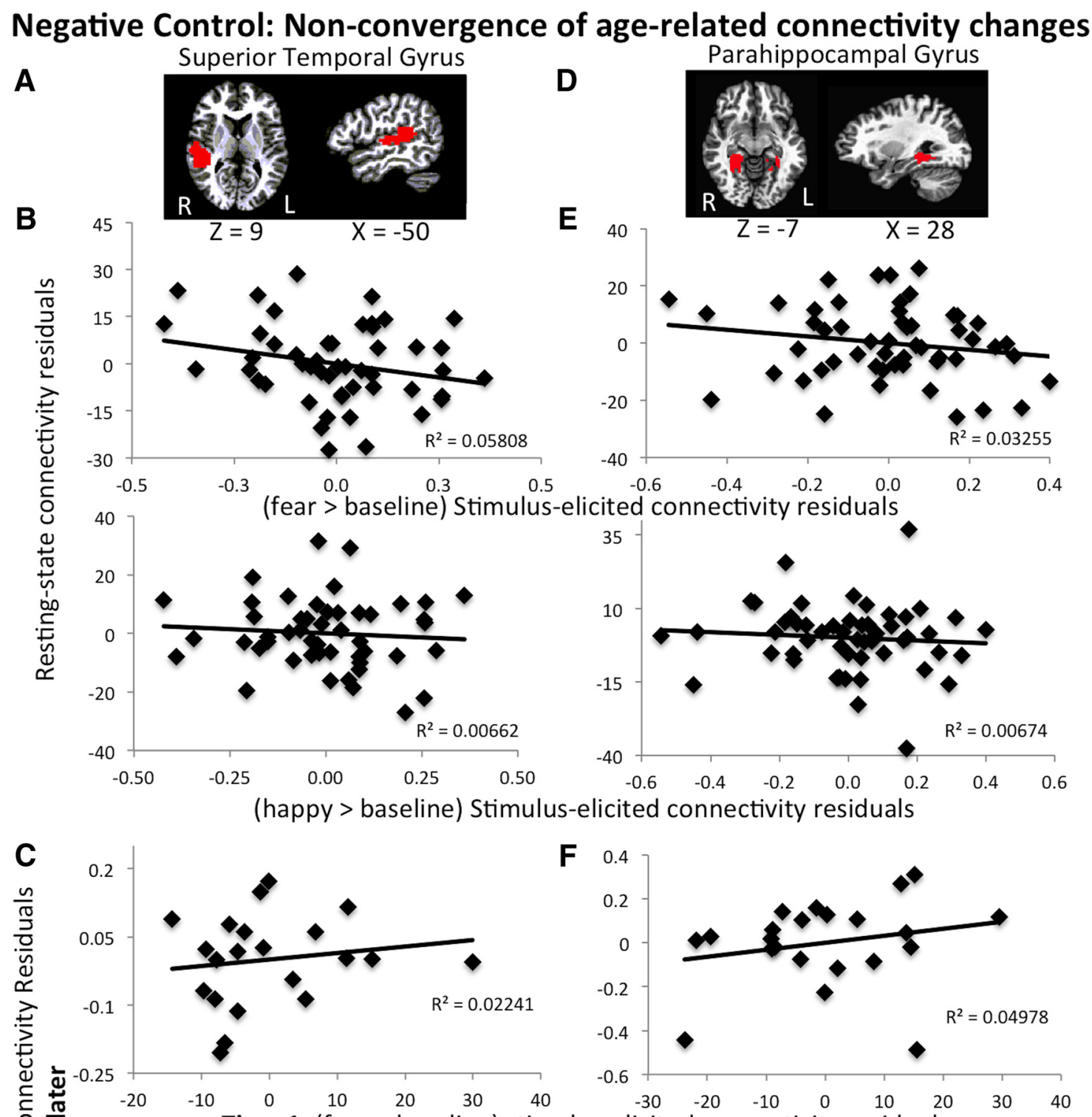

Time 1: (fear > baseline) stimulus-elicited connectivity residuals

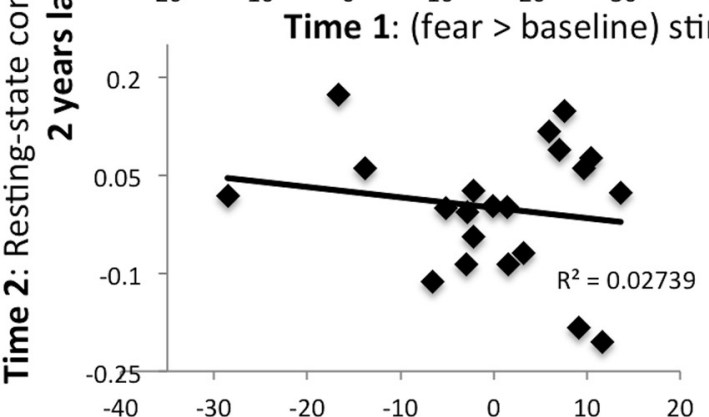

Time 1: (happy > baseline) stimulus-elicited connectivity residuals

Figure 6. Negative controls. Nonconvergence of age-related changes in connectivity patterns. $\boldsymbol{A}$, Area of right hemisphere superior temporal gyrus (STG) (Brodmann areas 41, 22) showing age-related changes in resting-state connectivity but no significant (or age-related changes in) stimulus-elicited connectivity in either fear or happy condition used to define amygdala-STG circuit. $\boldsymbol{B}$, Nonsignificant concurrent associations between residualized parameter estimates ( $\beta$ weights) for stimulus-elicited (fear condition, top row; happy condition, bottom row) and resting-state functional connectivities for the amygdala-STG circuit, controlling for age and motion covariates. $C$, Nonsignificant prospective associations between residualized parameter estimates ( $\beta$ weights) for stimulus-elicited connectivity at the first scan (fear condition, top row; happy condition, bottom row) and resting-state functional connectivity 2 years later for the STG-amygdala circuit, controlling for the exact time difference between scans, age, and resting-state connectivity estimates at the first scan. $\boldsymbol{D}$, Area of bilateral parahippocampal (PH) gyrus (Brodmann area 36) showing age-related changes in resting-state connectivity but no significant (or age-related changes in) stimulus-elicited connectivity in either fear or happy condition used to define amygdala-PH circuit. $\boldsymbol{E}$, Nonsignificant concurrent associations between residualized parameter estimates ( $\beta$ weights) for stimulus-elicited (fear condition, top row; happy condition, bottom row) and resting-state functional connectivities for the amygdala-PH circuit, controlling for age and motion covariates. $\boldsymbol{F}$, Nonsignificant prospective associations between residualized parameter estimates ( $\beta$ weights) for stimulus-elicited connectivity at the first scan (fear condition, top row; happy condition, bottom row) and resting-state functional connectivity 2 years later for the amygdala-PH circuit, controlling for the exact time difference between scans, age, and resting-state connectivity estimates at the first scan. 
hood and adolescence. These findings have several implications, discussed below, for our understanding of the ontogenetic mechanisms and plasticity of human resting-state connectivity emergence and its relation with stimulus-elicited functional connectivity measures across development and in maturity.

These results suggest that stimulus-elicited connectivity shapes the nature of resting-state connectivity through phasic molding occurring normatively on a developmental scale on the order of years, in support of the long-term phasic molding hypothesis. Specifically, these results show that the shaping process occurs throughout childhood, and in some cases into adolescence, for the amygdala-prefrontal functional network. The control analysis demonstrating an association between connectivity modalities maintained after a connection's stabilization is consistent with prior influence of stimulus-elicited connectivity on resting-state connectivity and provides evidence that a relation between connectivities persists in mature networks. Moreover, control analyses of nonconvergent age-related changes in connectivity further support the molding hypothesis by highlighting the specificity of our findings. That is, connections that do not show stimulus-elicited connectivity during the emotional faces paradigm also do not show any association between stimuluselicited connectivity estimates and the developing resting-state connectivity that is shaped by other unmeasured contexts. Thus, the observed associations between stimulus-elicited and restingstate connectivities do not seem to reflect global artifact (e.g., respiration artifact) but are specific to connections where the particular stimuli used in this study induce a phasic coordinated response.

Moreover, the developmental period during which stimuluselicited connectivity could influence future resting-state connectivity varied across amygdala-prefrontal connections and may indicate different trajectories of connection malleability. While stimuluselicited connectivity influenced amygdala-IFG resting-state connectivity across childhood and adolescence, for the amygdala-mPFC connection, we observed a developmental switch-point at the onset of adolescence after which stimulus-elicited connectivity did not influence future resting-state connectivity. This finding is consistent with adolescence demarcating the terminal boundary of a sensitive period for amygdala-mPFC functional circuitry (Fig. 2B). Further support for this interpretation comes from findings that children uniquely do not maintain stable resting-state coupling, indicative of increased malleability, and that their amygdala-mPFC circuit is uniquely sensitive to other environmental stimuli (e.g., the presence or absence of a parent) (Qin et al., 2012; Gabard-Durnam et al., 2014). Together, these results suggest that childhood demarcates a developmental period of exaggerated malleability (i.e., sensitive period) and thus increased potential for long-term shaping in the construction of the amygdala-mPFC circuit. However, stimulus-elicited amygdala-IFG connectivity influenced later resting-state connectivity across childhood and adolescence in this study. Notably, this region of the IFG has previously been implicated in cognitive control and affect reappraisal (coordinates overlap spatially with NeuroSynth automated meta-analyses), and both the IFG and these processes have been shown to have protracted developmental trajectories continuing into young adulthood (Casey et al., 2005; Lenroot and Giedd, 2006; Wager et al., 2008; Luna et al., 2010; McRae et al., 2012). Given the lengthy duration of IFG functional development, it is possible that our finding that amygdala-IFG stimulus-elicited connectivity continues to influence resting-state connectivity across adolescence reflects a more extended period of malleability for this connection relative to the amygdala-mPFC connection. Future work extending this assessment beyond age 18 years is necessary to determine whether the amygdala-IFG connection's malleability tapers off or persists through young adulthood.

Notably, initial adult studies exploring training effects on mature, established resting-state connectivity patterns suggest that resting-state connectivity may continue to undergo refinement through different, short-term Hebbian-like mechanisms in maturity (Albert et al., 2009; Lewis et al., 2009; Tambini et al., 2010; Harmelech et al., 2013; Mackey et al., 2013). Preliminary research of the Hebbian-like mechanisms in adults has suggested that reactivation of regions during sleep may facilitate this process in mature systems (Laureys et al., 2001; Harmelech et al., 2013). Sleep-based consolidation could be explored in future studies as a candidate means facilitating the developmental phasic molding of resting-state connectivity that occurs across years.

In addition, our direct comparison of resting-state connectivity with stimulus-elicited connectivity for the same amygdalaprefrontal network within the same individuals revealed the development of contrasting connectivity valences between stimulus and resting-state conditions. That is, in an adult individual, positive resting-state connectivity is associated with negative stimulus-elicited connectivity for the amygdala-mPFC circuit, and negative resting-state connectivity is associated with positive stimulus-elicited connectivity for the amygdala-IFG circuit. Preliminary research exploring stimulus-elicited activations and resting-state connectivity colocalization for mature networks, largely focused on the default-mode network, suggests that similar inverse relations may be observed (Thomason et al., 2008; Smith et al., 2009; Mennes et al., 2010, 2013)'. Attenuation of the inverse association between the default-mode network and stimulus-elicited activity has been associated with inefficient network function, poor task performance, and even a range of clinical phenotypes (Sonuga-Barke and Castellanos, 2007; Kelly et al., 2008; Mannell et al., 2010; Hamilton et al., 2011; Liddle et al., 2011). Inverse relationships between stimulus-elicited and resting-state connectivity valences may therefore generally mark efficient communication within networks (Buckner and Vincent, 2007; Tomasi et al., 2013; Guerra-Carrillo et al., 2014). However, whether our finding of oppositely valenced functional connectivities is a generalizable phenomenon needs to be further explored.

Given these results documenting the coemergence of functional connectivities for the amygdala-prefrontal network, it is important to examine whether these same mechanisms are at play in other functional networks emerging across development. Although we did not observe any predictive influence of resting-state connectivity on stimulus-elicited functional connectivity, it is possible that our small sample size precluded us from finding a weaker effect. Alternatively, it has been suggested that resting-state connectivity may constrain stimulus-based connectivity patterns in mature systems, and future studies extending the present age cap of 18 years into adulthood may capture a later developmental reversal in the direction of influence between functional connectivities (Deco and Corbetta, 2011). Future research relating to systems-level mechanisms of functional plasticity could help characterize the potential childhood period of malleability for the amygdala-mPFC circuit and evaluate potential factors triggering connectivity stabilization after childhood, including puberty onset, changes in hormone expression, or the psychosocial transition from elementary to middle school (Johansen-Berg, 2013).

In conclusion, our results provide critical insight into how the nature of the connectivity elicited by environmental events may shape resting-state connectivity across development and highlight childhood as a potential developmental period of heightened malle- 
ability for the amygdala-mPFC circuit. These findings suggest that early environmental events sculpt future functional network architecture, with implications for how both positive and adverse experiences influence the developing brain. Future research characterizing developmental periods of plasticity for amygdala-prefrontal circuits will be critical for understanding affective behaviors and when interventions for affective psychopathology can be most effective. Importantly, the neural systems-level mechanism we describe, whereby previous phasic experience influences the development of restingstate connectivity, is consistent with the single animal investigation comparing the development of phasic and resting activity at the neural population level within a visual cortex region in ferrets (Berkes et al., 2011). This concordance is especially striking given the different species, brain regions, and levels of neural processes involved. Accordingly, the present results indicate that this developmental long-term phasic modeling mechanism of circuit formation may be preserved across levels of neural complexity and motivate future investigations of whether this mechanism reflects a general phenomenon of brain development.

\section{References}

Achenbach TM, Edelbrock CS (1983) Manual for the Child Behavior Checklist and Revised Child Behavior Profile. Burlington, VT: University of Vermont, Department of Psychiatry.

Albert NB, Robertson EM, Miall RC (2009) The resting human brain and motor learning. Curr Biol 19:1023-1027. CrossRef Medline

Bandettini P, Smith SM (2012) The future of FMRI connectivity. Neuroimage 62:1257-1266. CrossRef Medline

Banks SJ, Eddy KT, Angstadt M, Nathan PJ, Phan KL (2007) Amygdalafrontal connectivity during emotion regulation. Soc Cogn Affect Neurosci 2:303-312. CrossRef Medline

Barber AD, Caffo BS, Pekar JJ, Mostofsky SH (2013) Developmental changes in within- and between-network connectivity between late childhood and adulthood. Neuropsychologia 51:156-167. CrossRef Medline

Berkes P, Orbán G, Lengyel M, Fiser J (2011) Spontaneous cortical activity reveals hallmarks of an optimal internal model of the environment. Science 331:83-87. CrossRef Medline

Buckner RL, Vincent JL (2007) Unrest at rest: default activity and spontaneous network correlations. Neuroimage 37:1091-1096; discussion 1097-1099. CrossRef Medline

Burgund ED, Kang HC, Kelly JE, Buckner RL, Snyder AZ, Petersen SE, Schlaggar BL (2002) The feasibility of a common stereotactic space for children and adults in fMRI studies of development. Neuroimage 17: 184-200. CrossRef Medline

Casey BJ, Galvan A, Hare TA (2005) Changes in cerebral functional organization during cognitive development. Curr Opin Neurobiol 15:239-244. CrossRef Medline

Chen G, Chen G, Xie C, Ward BD, Li W, Antuono P, Li SJ (2012) A method to determine the necessity for global signal regression in resting-state fMRI studies. Magn Reson Med 68:1828-1835. CrossRef Medline

Cohen MX, Heller AS, Ranganath C (2005) Functional connectivity with anterior cingulate and orbitofrontal cortices during decision-making. Brain Res Cogn Brain Res 23:61-70. CrossRef Medline

Cole DM, Smith SM, Beckmann CF (2010) Advances and pitfalls in the analysis and interpretation of resting-state FMRI data. Front Syst Neurosci 4:8. CrossRef Medline

Cox CL, Gotimer K, Roy AK, Castellanos FX, Milham MP, Kelly C (2010) Your resting brain CAREs about your risky behavior. PLoS One 5:e12296. CrossRef Medline

Cox RW (1996) AFNI: software for analysis and visualization of functional magnetic resonance neuroimages. Comput Biomed Res 29:162-173. CrossRef Medline

Decety J, Michalska KJ (2010) Neurodevelopmental changes in the circuits underlying empathy and sympathy from childhood to adulthood. Dev Sci 13:886-899. CrossRef Medline

Deco G, Corbetta M (2011) The dynamical balance of the brain at rest. Neuroscientist 17:107-123. CrossRef Medline

Di X, Gohel S, Kim EH, Biswal BB (2013) Task vs. rest-different network configurations between the coactivation and the resting-state brain networks. Front Hum Neurosci 7:493. CrossRef Medline
Dosenbach NUF, Fair DA, Miezin FM, Cohen AL, Wenger KK, Dosenbach RAT, Fox MD, Snyder AZ, Vincent JL, Raichle ME, Schlaggar BL, Petersen SE (2007) Distinct brain networks for adaptive and stable task control in humans. Proc Natl Acad Sci USA 104:11073-11078. CrossRef Medline

Friston KJ, Buechel C, Fink GR, Morris J, Rolls E, Dolan RJ (1997) Psychophysiological and modulatory interactions in neuroimaging. Neuroimage 6:218-229. CrossRef Medline

Gabard-Durnam LJ, Flannery J, Goff B, Gee DG, Humphreys KL, Telzer E, Hare T, Tottenham N (2014) The development of human amygdala functional connectivity at rest from 4 to 23 years: a cross-sectional study. Neuroimage 95:193-207. CrossRef Medline

Gee DG, Humphreys KL, Flannery J, Goff B, Telzer EH, Shapiro M, Hare TA, Bookheimer SY, Tottenham N (2013) A developmental shift from positive to negative connectivity in human amygdala-prefrontal circuitry. J Neurosci 33:4584-4593. CrossRef Medline

Gee DG, Gabard-Durnam L, Telzer EH, Humphreys KL, Goff B, Shapiro M, Flannery J, Lumian DS, Fareri DS, Caldera C, Tottenham N (2014) Maternal buffering of human amygdala-prefrontal circuitry during childhood but not during adolescence. Psychol Sci 25:2067-2078. CrossRef Medline

Greenough WT, Black JE, Wallace CS (1987) Experience and brain development. Child Dev 58:539-559. CrossRef Medline

Guerra-Carrillo B, Mackey AP, Bunge SA (2014) Resting-state fMRI: a window into human brain plasticity. Neuroscientist 20:522-533. CrossRef Medline

Hallquist MN, Hwang K, Luna B (2013) The nuisance of nuisance regression: spectral misspecification in a common approach to resting-state fMRI preprocessing reintroduces noise and obscures functional connectivity. Neuroimage 82:208-225. CrossRef Medline

Hamilton JP, Furman DJ, Chang C, Thomason ME, Dennis E, Gotlib IH (2011) Default-mode and task-positive network activity in major depressive disorder: implications for adaptive and maladaptive rumination. Biol Psychiatry 70:327-333. CrossRef Medline

Hare TA, Tottenham N, Galvan A, Voss HU, Glover GH, Casey BJ (2008) Biological substrates of emotional reactivity and regulation in adolescence during an emotional go-nogo task. Biol Psychiatry 63:927-934. CrossRef Medline

Harmelech T, Preminger S, Wertman E, Malach R (2013) The day-after effect: long term, Hebbian-like restructuring of resting-state fMRI patterns induced by a single epoch of cortical activation. J Neurosci 33: 9488-9497. CrossRef Medline

Johansen-Berg H (2013) Human connectomics: what will the future demand? Neuroimage 80:541-544. CrossRef Medline

Jolles DD, van Buchem MA, Crone EA, Rombouts SA (2011) A comprehensive study of whole-brain functional connectivity in children and young adults. Cereb Cortex 21:385-391. CrossRef Medline

Kang HC, Burgund ED, Lugar HM, Petersen SE, Schlaggar BL (2003) Comparison of functional activation foci in children and adults using a common stereotactic space. Neuroimage 19:16-28. CrossRef Medline

Keller CJ, Bickel S, Honey CJ, Groppe DM, Entz L, Craddock RC, Lado FA, Kelly C, Milham M, Mehta AD (2013) Neurophysiological investigation of spontaneous correlated and anticorrelated fluctuations of the BOLD signal. J Neurosci 33:6333-6342. CrossRef Medline

Kelly AM, Uddin LQ, Biswal BB, Castellanos FX, Milham MP (2008) Competition between functional brain networks mediates behavioral variability. Neuroimage 39:527-537. CrossRef Medline

Kim MJ, Gee DG, Loucks RA, Davis FC, Whalen PJ (2011) Anxiety dissociates dorsal and ventral medial prefrontal cortex functional connectivity with the amygdala at rest. Cereb Cortex 21:1667-1673. CrossRef Medline

Laureys S, Peigneux P, Phillips C, Fuchs S, Degueldre C, Aerts J, Del Fiore G, Petiau C, Luxen A, van der Linden M, Cleeremans A, Smith C, Maquet P (2001) Experience-dependent changes in cerebral functional connectivity during human rapid eye movement sleep. Neuroscience 105:521-525. CrossRef Medline

Lenroot RK, Giedd JN (2006) Brain development in children and adolescents: insights from anatomical magnetic resonance imaging. Neurosci Biobehav Rev 30:718-729. CrossRef Medline

Lewis CM, Baldassarre A, Committeri G, Romani GL, Corbetta M (2009) Learning sculpts the spontaneous activity of the resting human brain. Proc Natl Acad Sci U S A 106:17558-17563. CrossRef Medline

Liddle EB, Hollis C, Batty MJ, Groom MJ, Totman JJ, Liotti M, Scerif G, 
Liddle PF (2011) Task-related default mode network modulation and inhibitory control in ADHD: effects of motivation and methylphenidate. J Child Psychol Psychiatry 52:761-771. CrossRef Medline

Luna B, Padmanabhan A, O’Hearn K (2010) What has fMRI told us about the development of cognitive control through adolescence? Brain Cogn 72:101-113. CrossRef Medline

Lundqvist D, Flykt A, Öhman A (1998) The Karolinska directed emotional faces (KDEF). CD ROM from Department of Clinical Neuroscience, Psychology section, Karolinska Institutet, 91-630.

Mackey AP, Miller Singley AT, Bunge SA (2013) Intensive reasoning training alters patterns of brain connectivity at rest. J Neurosci 33:4796-4803. CrossRef Medline

MacNamara A, DiGangi J, Phan KL (2015) Aberrant spontaneous and taskdependent functional connections in the anxious brain. Biol Psychiatry Cogn Neurosci Neuroimaging. Advance Online Publication. Retrieved April 6, 2016. doi: 10.1016/j.bpsc.2015.12.004.

Mannell MV, Franco AR, Calhoun VD, Cañive JM, Thoma RJ, Mayer AR (2010) Resting state and task-induced deactivation: a methodological comparison in patients with schizophrenia and healthy controls. Hum Brain Mapp 31:424-437. CrossRef Medline

McRae K, Gross JJ, Weber J, Robertson ER, Sokol-Hessner P, Ray RD, Gabrieli JD, Ochsner KN (2012) The development of emotion regulation: an fMRI study of cognitive reappraisal in children, adolescents and young adults. Soc Cogn Affect Neurosci 7:11-22. CrossRef Medline

Mennes M, Kelly C, Zuo XN, Di Martino A, Biswal BB, Castellanos FX, Milham MP (2010) Inter-individual differences in resting-state functional connectivity predict task-induced BOLD activity. Neuroimage 50: 1690-1701. CrossRef Medline

Mennes M, Kelly C, Colcombe S, Castellanos FX, Milham MP (2013) The extrinsic and intrinsic functional architectures of the human brain are not equivalent. Cereb Cortex 23:223-229. CrossRef Medline

Miall RC, Robertson EM (2006) Functional imaging: is the resting brain resting? Curr Biol 16:R998-R1000. CrossRef Medline

Perlman SB, Pelphrey KA (2011) Developing connections for affective regulation: age-related changes in emotional brain connectivity. J Exp Child Psychol 108:607-620. CrossRef Medline

Pizoli CE, Shah MN, Snyder AZ, Shimony JS, Limbrick DD, Raichle ME, Schlaggar BL, Smyth MD (2011) Resting-state activity in development and maintenance of normal brain function. Proc Natl Acad Sci U S A 108:11638-11643. CrossRef Medline

Power JD, Schlaggar BL, Petersen SE (2014) Recent progress and outstanding issues in motion correction in resting state fMRI. Neuroimage 105: 536-551. CrossRef Medline

Qin S, Young CB, Supekar K, Uddin LQ, Menon V (2012) Immature integration and segregation of emotion-related brain circuitry in young children. Proc Natl Acad Sci U S A 109:7941-7946. CrossRef Medline

Raichle ME (2010) Two views of brain function. Trends Cogn Sci 14: 180-190. CrossRef Medline

Roy AK, Shehzad Z, Margulies DS, Kelly AM, Uddin LQ, Gotimer K, Biswal BB, Castellanos FX, Milham MP (2009) Functional connectivity of the human amygdala using resting state fMRI. Neuroimage 45:614-626. CrossRef Medline

Satterthwaite TD, Wolf DH, Loughead J, Ruparel K, Elliott MA, Hakonarson H, Gur RC, Gur RE (2012) Impact of in-scanner head motion on multiple measures of functional connectivity: relevance for studies of neurodevelopment in youth. Neuroimage 60:623-632. CrossRef Medline
Siegel JS, Power JD, Dubis JW, Vogel AC, Church JA, Schlaggar BL, Petersen SE (2014) Statistical improvements in functional magnetic resonance imaging analyses produced by censoring high-motion data points. Hum Brain Mapp 35:1981-1996. CrossRef Medline

Smith SM, Fox PT, Miller KL, Glahn DC, Fox PM, Mackay CE, Filippini N, Watkins KE, Toro R, Laird AR, Beckmann CF (2009) Correspondence of the brain's functional architecture during activation and rest. Proc Natl Acad Sci U S A 106:13040-13045. CrossRef Medline

Solé-Padullés C, Castro-Fornieles J, de la Serna E, Calvo R, Baeza I, Moya J, Lázaro L, Rosa M, Bargalló N, Sugranyes G (2016) Intrinsic connectivity networks from childhood to late adolescence: effects of age and sex. Dev Cogn Neurosci 17:35-44. CrossRef Medline

Sonuga-Barke EJ, Castellanos FX (2007) Spontaneous attentional fluctuations in impaired states and pathological conditions: a neurobiological hypothesis. Neurosci Biobehav Rev 31:977-986. CrossRef Medline

Swartz JR, Carrasco M, Wiggins JL, Thomason ME, Monk CS (2014) Agerelated changes in the structure and function of prefrontal cortexamygdala circuitry in children and adolescents: a multi-modal imaging approach. Neuroimage 86:212-220. CrossRef Medline

Talairach J, Tournoux P (1988) Co-planar stereotaxic atlas of the human brain: 3-dimensional proportional system-an approach to cerebral imaging. Stuttgart and New York: Thieme Verlag.

Tambini A, Ketz N, Davachi L (2010) Enhanced brain correlations during rest are related to memory for recent experiences. Neuron 65:280-290. CrossRef Medline

Telzer EH, Flannery J, Shapiro M, Humphreys KL, Goff B, Gabard-Durman L, Gee DD, Tottenham N (2013) Early experience shapes amygdala sensitivity to race: an international adoption design. J Neurosci 33: 13484-13488. CrossRef Medline

Thomason ME, Chang CE, Glover GH, Gabrieli JD, Greicius MD, Gotlib IH (2008) Default-mode function and task-induced deactivation have overlapping brain substrates in children. Neuroimage 41:1493-1503. CrossRef Medline

Tomasi D, Wang GJ, Volkow ND (2013) Energetic cost of brain functional connectivity. Proc Natl Acad Sci U S A 110:13642-13647. CrossRef Medline

Uddin LQ, Supekar KS, Ryali S, Menon V (2011) Dynamic reconfiguration of structural and functional connectivity across core neurocognitive brain networks with development. J Neurosci 31:18578-18589. CrossRef Medline

Van Dijk KR, Hedden T, Venkataraman A, Evans KC, Lazar SW, Buckner RL (2010) Intrinsic functional connectivity as a tool for human connectomics: theory, properties, and optimization. J Neurophysiol 103:297-321. CrossRef Medline

Van Dijk KR, Sabuncu MR, Buckner RL (2012) The influence of head motion on intrinsic functional connectivity MRI. Neuroimage 59:431-438. CrossRef Medline

Wager TD, Nichols TE (2003) Optimization of experimental design in fMRI: a general framework using a genetic algorithm. Neuroimage 18: 293-309. CrossRef Medline

Wager TD, Davidson ML, Hughes BL, Lindquist MA, Ochsner KN (2008) Prefrontal-subcortical pathways mediating successful emotion regulation. Neuron 59:1037-1050. CrossRef Medline

Yan CG, Cheung B, Kelly C, Colcombe S, Craddock RC, Di Martino A, Li Q, Zuo XN, Castellanos FX, Milham MP (2013) A comprehensive assessment of regional variation in the impact of head micromovements on functional connectomics. Neuroimage 76:183-201. CrossRef Medline 